\title{
Aneurisma dissecante de aorta tipo A de Stanford com extensão às ilíacas: um relato de caso
}

\section{Stanford type A dissecting aortic aneurysm with iliac extension: a case report}

Thalita Cavalcanti Muliterno das Neves ${ }^{1 *}$, Alana Carla Albuquerque Sarmento ${ }^{l}$, Bárbara Souza Leão Santiago ${ }^{l}$, Esthefany Dias Barbosa ${ }^{I}$, Ingrid Cardoso Cipriano ${ }^{I}$, Isadora Veiga de Albuquerque ${ }^{l}$, João Emanuel do Nascimento ${ }^{1}$, Marcos Luan Ribeiro do Nascimento ${ }^{\prime}$, Nathália Almeida Vieira de Mello ${ }^{1}$, Osvaldo Carlos Rodrigues Júnior ${ }^{1}$, Regina Maria Lima Braga da Silval.

${ }^{1}$ Hospital Universitário Oswaldo Cruz, Universidade de Pernambuco, Recife, Brasil.

*correspondência: thalita.cm@hotmail.com

Recebido: 30/10/2019. Aceito: 12/11/2019.

\section{Resumo}

O presente relato descreve o caso de um paciente diagnosticado há 3 anos com aneurisma de aorta, que chega à emergência com dissecção da aorta ascendente até as artérias ilíacas evidenciada por angiotomografia computadorizada. Apesar da ausência de sintomas à admissão, devido ao alto índice de mortalidade do aneurisma dissecante, foi indicada abordagem cirúrgica. Optou-se pelo procedimento de Bentall-De Bono para correção de dissecção de raiz da aorta e insuficiência aórtica. Os autores ressaltam a importância do diagnóstico precoce e da terapia efetiva para a redução da morbimortalidade.

Palavras-chave: aorta; aorta/cirurgia; Aneurisma dissecante/cirurgia; Cirurgia de Bentall; dor torácica.

\begin{abstract}
This report describes the case of a patient diagnosed with chronic aortic aneurysm in the past 3 years, who arrived at the emergency room with a dissection of the ascending aorta to the iliac arteries detected by computed tomography angiography. Despite the absence of symptoms at admission, due to the high mortality rate of the dissecting aneurysm, it was indicated surgery approach. The chosen procedure was a Bentall-De Bono procedure to correct the aortic root dissection and aortic insufficiency. The authors emphasize the importance of early diagnosis and effective therapy for the reduction of morbidity and mortality.
\end{abstract}

Key words: aorta; aorta/surgery; aneurysm, dissecting/surgery; Bentall procedure; thoracic pain.

\section{Introdução}

A dissecção de aorta consiste em uma delaminação da parede da artéria que favorece a penetração de sangue pela separação das camadas íntima e média, levando a formação de dois lúmens, um falso e um verdadeiro. Muitas vezes decorre da sobrecarga pressórica exercida sobre uma área dilatada, ou seja, aneurismática. Configura-se aneurisma uma dilatação arterial maior que 50\% do diâmetro normal do vaso que apresenta elevada morbimortalidade, principalmente quando seu manejo não é realizado de forma adequada. ${ }^{1}$

Os aneurismas do arco aórtico e da aorta torácica descendente têm em sua gênese a aterosclerose, sendo considerados degenerativos. Os de aorta ascendente, por sua vez, estão diretamente relacionados à necrose cística da camada média, um distúrbio que predispõe a dissecção. Os segmentos mais acometidos incluem o abdominal infrarrenal (80\%), o torácico descendente $(12 \%)$ e o torácico ascendente $(5,5 \%)$. Sem tratamento, 75 a $80 \%$ dos aneurismas aórticos rompem em uma média de 2 a 3 anos após o diagnóstico. ${ }^{1,2}$

Dissecção aórtica é rara em pacientes com menos de 40 anos de idade, a menos que esteja associada a uma síndrome de predisposição específica, como Síndrome de Marfan, EhlersDanlos, doença cardíaca congênita, coarctação da 
aorta, doença de Turner, o uso de cocaína ou trauma. A hipertensão arterial é a causa mais comum e é detectada em mais da metade dos pacientes acometidos por essa afecção. Ocorrem entre 10 a 20 casos por milhão de habitantes por ano e, se não tratada adequadamente, a mortalidade é de cerca de $50 \%$ em 48 horas e de 60 a $90 \%$ em uma semana. ${ }^{1-3}$

No presente relato, os autores descrevem uma dissecção de aorta do tipo A de Stanford em um paciente com aneurisma aórtico, submetido à cirurgia de Bentall-De Bono no Pronto Socorro Cardiológico de Pernambuco - Prof. Luiz Tavares (PROCAPE).

\section{Relato de caso}

Paciente do sexo masculino, 52 anos, que chega a emergência do PROCAPE com diagnóstico angiotomográfico computadorizado de aneurisma dissecante de aorta do tipo A com extensão até as artérias ilíacas, estando assintomático na admissão. Há 5 anos do internamento, iniciou episódios esporádicos de dor torácica em pontada, com duração de poucos segundos e intensidade 8 (sic), numa escala de 0 a 10. Há 3 anos, iniciou investigação médica, na qual evidenciou, ao ecocardiograma, dilatação de aorta, sendo acompanhado semestralmente por exames de imagem, sem relato de mudança no padrão da dor.

Como antecedentes apresentava hipertensão arterial sistêmica, obesidade, diabetes mellitus tipo II, dislipidemia e gota, em uso ambulatorial de losartana, succinato de metoprolol, cloridrato de clonidina, sinvastatina e ácido acetilsalicílico.

Ao exame inicial no PROCAPE, o paciente apresentou estado geral bom, pressão arterial em membro superior direito de $170 \times 60 \mathrm{mmHg}$ e em membro superior esquerdo de $150 \times 70 \mathrm{mmHg}$, frequência cardíaca de 96 batimentos por minuto; pulsos cheios e simétricos; a ausculta cardíaca evidenciou sopro diastólico em borda esternal direita alta $(3+/ 6+)$, com irradiação para fúrcula esternal. Os exames dos aparelhos respiratório e digestivo não apresentavam alterações. $\mathrm{O}$ eletrocardiograma evidenciou ritmo sinusal regular e complexo QRS sem alterações.

Uma semana antes do internamento foi realizado ecocardiograma que constatou fração de ejeção do ventrículo esquerdo preservada (74\%), importante dilatação da aorta ascendente $(6,5 \mathrm{~cm})$ e insuficiências valvares aórtica de grau moderado e mitral de grau leve. Diante dos achados, realizou-se angiotomografia computadorizada (Figura 1), que evidenciou área cardíaca aumentada globalmente, ectasia difusa da aorta torácica, dissecção com início no seio de Valsalva, percorrendo a aorta ascendente e se estendendo para o segmento abdominal até a bifurcação; há extensão da dissecção na artéria ilíaca direita e no tronco braquiocefálico. Observou-se um diâmetro transverso da aorta torácica ascendente de $6,6 \mathrm{~cm}$; uma dilatação fusiforme com diâmetro transverso de $5,8 \mathrm{~cm}$ no segmento torácico inferior e um diâmetro de $4,3 \mathrm{~cm}$ no segmento abdominal infrarrenal. Nos dois últimos segmentos, havia presença de trombo mural.

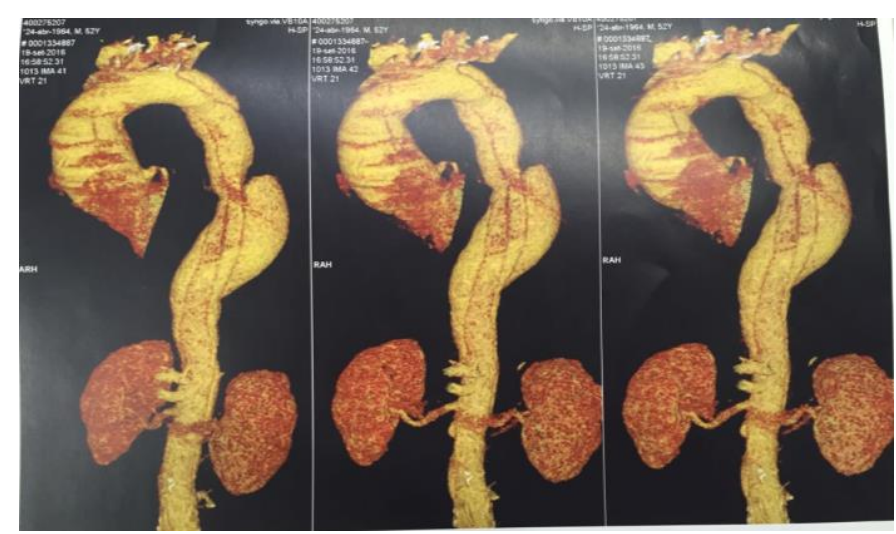

Figura 1. Reconstrução vascular de angiotomografia computadorizada evidenciando dilatação aneurismática da aorta e dissecção em porção ascendente, descendente e abdominal.

Após cuidados iniciais, incluindo administração de nitroprussiato de sódio e succinato de metoprolol, o paciente foi encaminhado para a realização da cirurgia de Bentall-De Bono, com implante no anel aórtico de tubo valvulado de Ddacron e de válvula de pericárdio bovino $(\mathrm{PB}) \mathrm{N}^{\mathrm{o}}$ 25.

O paciente evoluiu no pós-operatório com infecção nosocomial do trato respiratório e colite pseudomembranosa, devidamente tratadas. Também apresentou trombose de veia axilar esquerda, relacionada ao cateter venoso central instalado na veia subclávia esquerda, sendo conduzido com anticoagulação oral. Após o $26^{\circ}$ dia de pósoperatório, recebeu alta hospitalar com bom estado geral e em uso de sinvastatina, succinato de 
metoprolol, paracetamol + codeína, enalapril, clonazepam, rivaroxabana e pantoprazol sódico.

\section{Discussão}

A etiologia do aneurisma dissecante é complexa e multifatorial, destacando-se como principais fatores de risco: tabagismo, aterosclerose, altos níveis pressóricos, doenças degenerativas da aorta, diâmetro aórtico superior a $5 \mathrm{~cm}$ e anomalias cardiovasculares congênitas. ${ }^{4}$ No caso descrito, o paciente apresentava hipertensão e dislipidemias como principais comorbidades associadas ao surgimento da doença vascular.

As dissecções de aorta (DA) são emergências hipertensivas, uma vez que representam condições clínicas caracterizadas pelo aumento progressivo da pressão arterial associado a lesões de órgãos-alvo, constituindo ameaça imediata à vida. ${ }^{5}$ Essas anormalidades são classificadas quanto ao tempo de apresentação em agudas quando até 14 dias, subagudas, de 15 a 90 dias, e crônicas, acima de 90 dias. Anatomicamente, a dissecção aórtica classifica-se em tipos A e B de Stanford, para implicações prognósticas e terapêuticas. As dissecções do tipo A são aquelas que abrangem a aorta ascendente e as do tipo B acometem apenas a aorta descendente. ${ }^{6}$

A DA do tipo A representa a lesão cardiovascular de maior mortalidade se não tratada de maneira imediata. Em sua fase aguda, $3 \%$ dos pacientes evoluem para morte súbita e, se não manejados a curto prazo, a mortalidade pode aumentar $1 \%$ por hora, nas primeiras 24 horas, sendo que $80 \%$ vão a óbito nas primeiras duas semanas e $95 \%$ em um ano. ${ }^{5,7}$

Clinicamente, a DA é marcada pela dor torácica de forte intensidade e súbito aparecimento. Tal dor pode ter localização variada, às vezes irradiando para o dorso e/ou abdome, a depender da região afetada pela dissecção. ${ }^{1}$ Neste relato, o paciente apresentou um quadro atípico, referindo desconforto torácico não limitante e de evolução crônica. Ao exame físico, pode haver a constatação de assimetria de pulsos, sinais de tamponamento cardíaco, insuficiência aórtica, atrito pericárdico e alterações neurológicas centrais ou periféricas por envolvimento da carótida. Este paciente apresentouse com pressões assimétricas nos membros superiores, além de insuficiência aórtica cuja fisiopatologia relaciona-se a dilatação sino-tubular e/ou distorção/dilatação dos seios de Valsalva e perda da sustentação de comissura e folhetos. ${ }^{8}$

Entre os exames subsidiários para o diagnóstico incluem-se ecocardiografia e angiotomografia, sendo esta o padrão ouro, com excelente definição espacial e sensibilidade e especificidades acima de $90 \%$. Podem ser utilizadas também radiografia, ressonância magnética e aortografia. ${ }^{9}$ Esses exames, além de estabelecerem o diagnóstico, visam dar informações anatômicas precisas para o seguimento da conduta. $\mathrm{Na}$ avaliação do caso, a angiotomografia apresenta achados indicativos de lesões agudas com componentes de lesões crônicas (Figura 2). Dentre os agudos, observa-se a existência de contraste em luz vascular percorrendo o caminho da dissecção e hematoma fusiforme em segmento torácico; dentre as características crônicas observa-se a presença de trombos e calcificações dentro do lúmen criado pela dissecção aórtica.

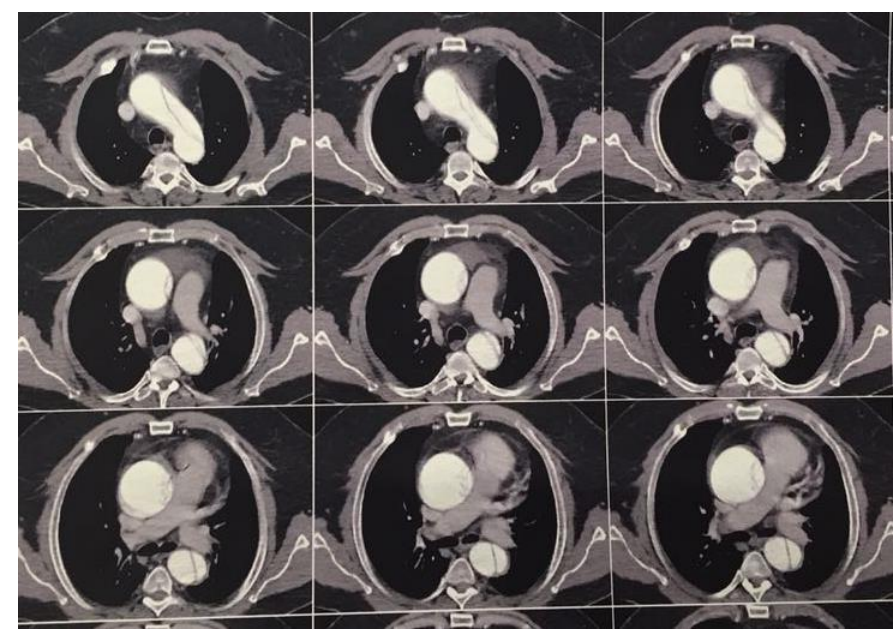

Figura 2. Angiotomografia evidenciando dilatação aneurismática importante da aorta torácica, com dissecção em ramos ascendente e descendente e visualização de trombo intramural.

A história natural do tipo A é desfavorável com tratamento clínico, ocorrendo complicações precoces como óbito por ruptura da aorta, insuficiência aórtica ou oclusão dos óstios coronários, sendo a conduta cirúrgica, a medida mais adequada. ${ }^{1,8}$ Assim, o paciente foi submetido à cirurgia de Bentall-De Bono para correção de dissecção de raiz da aorta e insuficiência aórtica, baseado nos achados da sua angiotomografia $\mathrm{e}$ 
ecocardiografia. Nas dissecções envolvendo aorta ascendente a cirurgia deverá ser imediata, no intuito de evitar ruptura e morte por tamponamento cardíaco, corrigir a insuficiência aórtica quando presente, evitar isquemia miocárdica, excluir o local da laceração da íntima e redirecionar o fluxo pela luz verdadeira. Quando a dissecção aguda ocorre em uma aorta ascendente e/ou raiz de aorta previamente dilatada a correção cirúrgica requer a substituição da válvula aórtica e reimplante dos óstios coronários. ${ }^{7,8,9}$

Foram realizadas, no caso relatado, esternotomia mediana e valvuloplastia com implante no anel aórtico de tubo valvulado de dacron com válvula $\mathrm{PB} \mathrm{N}^{\mathrm{o}} 25$ com pontos separados. Posteriormente, houve uma desinserção dos óstios coronarianos e reimplante na prótese com sutura contínua, seguido de anastomose da porção distal do tubo de pericárdio da aorta ascendente ao tronco braquiocefálico. Tal técnica trouxe diversas vantagens como diminuição dos tempos cirúrgico e de sangramento. Os achados intra-operatórios foram: grande rotura da íntima logo acima dos óstios coronarianos e sinais de dissecção crônica no óstio direito.

As possíveis complicações imediatas do procedimento Bentall-De Bono são sangramento, acidente vascular cerebral e infarto agudo do miocárdio, já as tardias são fístula aorto-átrio direito, estenose bilateral dos óstios coronarianos, pseudo-aneurisma, endocardite e tromboembolismo. ${ }^{10}$ No paciente em questão, não houve intercorrências cirúrgicas.

\section{Conclusão}

Diante do exposto, torna-se importante o diagnóstico precoce para uma conduta adequada que está atrelada a um menor índice de complicações. É necessário considerar diagnósticos diferenciais e manifestações clínicas atípicas, a fim de garantir uma assistência eficaz.

\section{Referências}

1. Serrano Júnior, CV, Timerman A, Stefanini E (2009) Tratado de Cardiologia da SOCESP, $2^{\mathrm{a}}$ ed, São Paulo, Manole.

2. Lederle FA (2009) In the clinic. Abdominal aortic aneurysm. Ann Intern Med 150:9.

3. Kouchoukos NT, Dougenis D (1997) Surgery of the thoracic aorta. N Engl J Med 336:(26)1876-1889.

4. Goldfinger JZ. et al. (2014) Thoracic aortic aneurysm and dissection. J Am Coll Cardiol 64:(16)1725-1739.

5. Silva, MAM et al. (2013) Crise hipertensiva, pseudocrise hipertensiva e elevação sintomática da pressão arterial. Rev Bras Cardiol, 26:(5)329-336.

6. Rolim D, Sampaio S, Dias PG, Almeida P, Ferreira RC, Teixeira JF (2016) Aneurisma toracoabdominal pós-dissecção crônica tipo B: um desafio anatômico com uma solução endovascular inesperadamente simples. Angiol Cir Vasc 12:(1)50-54.

7. Pontes JCDV; Duarte JJ, Silva AD; Dias AMAS (2012) Correção endovascular de aneurisma de aorta abdominal em complicação tardia de dissecção de aorta tipo A. Rev Bras Cir Cardiovasc 27:(4)645-647.

8. Pereira WM et al. (1997) Tratamento cirúrgico da insuficiência aórtica nos aneurismas e dissecções da aorta ascendente pela técnica de Tirone David. Braz J Cardiovasc Surg 12:(1)24-29.

9. Erbel R et al. (2001) Task force report. Eur Heart J 22:(18)1642-1681.

10. Albuquerque LC, Braile DM, Palma JH, Saadi EK, Almeida RMS (2009) Diretrizes para o tratamento cirúrgico das doenças da aorta da Sociedade Brasileira de Cirurgia Cardiovascular-Atualização 2009. Rev Bras Cir Cardiovasc 24:(2). 